\title{
Dynamics of states in the nonlinear interaction regime between a three-level atom and generalized coherent states and their non-classical features
}

\author{
M K Tavassoly ${ }^{1,2}$, F. Yadollahi ${ }^{1,3}$ \\ 1- Department of Mathematical Sciences, Yazd University, Yazd, Iran \\ 2- Research Group of Optics and Photonics, Yazd University, Yazd, Iran \\ 3- Department of Physics, Marvdasht branch, Islamic Azad University, Marvdasht, Iran \\ E-mail: mktavassoly@yazduni.ac.ir; ftm.yadollahi@yahoo.com
}

July 5, 2018

\begin{abstract}
The present study investigates the interaction of an equidistant three-level atom and a single-mode cavity field that has been initially prepared in a generalized coherent state. The atom-field interaction is considered to be, in general, intensitydependent. We suppose that the nonlinearity of the initial generalized coherent state of the field and the intensity-dependent coupling between atom and field are distinctly chosen. Interestingly, an exact analytical solution for the time evolution of the state of atom-field system can be found in this general regime in terms of the nonlinearity functions. Finally, the presented formalism has been applied to a few known physical systems such as Gilmore-Perelomov and Barut-Girardello coherent states of $S U(1,1)$ group, as well as a few special cases of interest. Mean photon number and atomic population inversion will be calculated, in addition to investigating particular non-classicality features such as revivals, sub-Poissonian statistics and quadratures squeezing of the obtained states of the entire system. Also, our results will be compared with some of the earlier works in this particular subject.
\end{abstract}

Keywords: Atom-field interaction; Nonlinear Jaynes-Cummings model (JCM); Nonlinear coherent state; Nonclassical state.

Pacs: 42.50.Ct, 42.50.Dv, 42.50.Ar, 42.50.-p 


\section{Introduction}

Coherent states (CSs) play an important role in quantum optics and modern physics [1, 2, 3]. Along different kinds of generalization of coherent states [5], nonlinear CSs [6] or $f$-CSs $|\alpha, f\rangle$ [7] have been introduced and attracted much attention in recent decade [8]-[13] with the Fock space representation as [7]:

$$
|\alpha, f\rangle=\left(\sum_{n=0}^{\infty} \frac{|\alpha|^{2 n}}{n !([f(n)] !)^{2}}\right)^{-1 / 2} \sum_{n=0}^{\infty} \frac{\alpha^{n}}{\sqrt{n !}[f(n)] !}|n\rangle, \quad \alpha=|\alpha| e^{i \varphi},
$$

where $[f(n)] ! \doteq f(1) f(2) \ldots f(n)$. According to this formalism, $f$-deformed annihilation and creation operators, respectively are defined as $A=a f(n)$ and so $A^{\dagger}=f^{\dagger}(n) a^{\dagger}$ where $a, a^{\dagger}$ and $n=a^{\dagger} a$ are bosonic annihilation, creation and number operator, respectively. Here, the (real) intensity-dependent function $f(n)$ is responsible for the nonlinearity of the states. On the other side, Jaynes-Cummings model (JCM) is the simplest nontrivial example of the atom-field interaction, i.e., a two-level atom and a single-mode radiation field [14]. As a few recent works in this topics see Refs. [15, 16]. The dynamical behaviour of the nonlinear atom-field interaction in the presence of classical gravity using the nonlinear coherent states approach discussed in [17]. Buzek generalized the JCM [18] and the atom-field coupling is considered to be intensity-dependent and supposed that the cavity field be in the Gilmore-Perelomov (GP) nonlinear CS of $S U(1,1)$ group [19]. The author showed that, the revivals of the radiation squeezing are strictly periodical for any value of initial squeezing parameter [18]. More recently, Koroli et al [20] studied the interaction of an equidistant three-level atom (ion), whose dipole moment matrix transition elements between the adjacent atomic levels are different, with the GP CS of $S U(1,1)$ group. They showed that, in the three-level model with the intensity-dependent coupling, the exact periodicity of the squeezing revivals is violated.

In the present paper, due to the great interest in the atom-field interactions in the quantum optics, we regard the same configuration of three-level atom (ion) has been considered in [20], however we generalize the initial state of the field to the "nonlinear CS" with arbitrary "nonlinearity function" $f(n)$. In our formalism, the atom-field coupling is also considered to be intensity-dependent, characterized by a function $g(n)$, which is generally different from $f(n)$. While in Refs. [18, 20] the authors have concerned with special nonlinearities, our presentation deals, in principle, with two distinct general nonlinearity functions and interestingly, the exact solution is also obtained for the time evolved entire states of the system.

After finding the explicit solution of the state vector, which evidently depends on $f(n)$ and $g(n)$, we will treat the time evolution of the mean photon number and atomic population inversion, as well as a few non-classicality features such as sub-Poissonian statistics and squeezing of the quadratures of the field. Therefore, in contrast to the approach of [20], that investigated only a special system, i.e., Holstein-Primakoff $S U(1,1)$ coherent 
states as filed state and $\sqrt{n}$ as the coupling between atom and field, our formalism can be applied to the intensity-dependent interaction between the same three-level atom with arbitrary cavity field that is initially prepared in a generalized CS with known "nonlinearity function $f(n)$ ". Interestingly, apart from the mentioned advantage, as we will observe, our presented formalism allows one to work with the general case in which two different nonlinearity functions exist, one indicates the initial state of the field $(f(n))$, and the other to the atom-field intensity dependent coupling $(g(n))$. Although, it can simply recover the work of Buzek [18] and Koroli et al [20], as some special cases. In the continuation, the formalism has been applied to two classes of generalized CSs, i.e., two distinct representations of $S U(1,1)$ CSs. In addition, in opposite of the work has been done in [20], in which the authors have taken $f(n)=1 / \sqrt{n}$ and $g(n)=\sqrt{n}$, we choose $f(n)=g(n)=\sqrt{n}$, i.e., the nonlinearity of the initial state is taken to be the same as the intensity-dependent coupling function. Finally, in each of the considered cases, we discuss the numerical results have been shown in several figures in detail and compare with earlier works. Along this procedure, some new physical features reveals, which may be emphasized. For instance, a typical collapse and revival phenomenon (as a pure quantum mechanical feature) in physical quantities such as mean photon number, atomic population inversion and Mandel parameter, and the appearance of some non-classicality signs, are specific aspects may be highlighted.

\section{Hamiltonian of atom-field system}

We consider the interaction of an equidistant three-level atom in a cascade configuration with different dipole moment matrix transition elements between the adjacent atomic energy levels (shown in Figure 1) with a quantized single-mode cavity field of frequency $\omega$. The states $|g\rangle,\left|e_{1}\right\rangle$ and $\left|e_{2}\right\rangle$ are respectively denoted the ground, first and second excited states. Only the atomic transitions between $|g\rangle,\left|e_{1}\right\rangle$ and also $\left|e_{1}\right\rangle,\left|e_{2}\right\rangle$ are dipole allowed, but not between $|g\rangle,\left|e_{2}\right\rangle$. The Hamiltonian of such an atom-field system is given by:

$$
H=H_{0}+H_{1}
$$

where $H_{0}$ is the sum of the Hamiltonians of atom and field, i.e., $H_{0}=\hbar \omega_{0} S_{z}+\hbar \omega a^{\dagger} a$, $S_{z}=\left|e_{2}\right\rangle\left\langle e_{2}|-| g\right\rangle\langle g|$, denotes the atomic population inversion operator. The interaction Hamiltonian between atom and field in (2) in the dipole and rotating wave approximation is given by:

$$
H_{1}=\hbar \lambda_{1}\left(a^{\dagger}\left|e_{1}\right\rangle\left\langle e_{2}|+a| e_{2}\right\rangle\left\langle e_{1}\right|\right)+\hbar \lambda_{2}\left(a^{\dagger}|g\rangle\left\langle e_{1}|+a| e_{1}\right\rangle\langle g|\right),
$$

where $\lambda_{1}, \lambda_{2}$ are the atom-field coupling constants. A useful approach to the atom-field interaction problem may be found in the "interaction picture" [21]. The Hamiltonian describing the interaction between the mentioned atom-field, in the interaction picture, 
is described by

$$
H_{I}=\hbar \lambda_{1}\left(a^{\dagger}\left|e_{1}\right\rangle\left\langle e_{2}\left|e^{-i \Delta t}+a\right| e_{2}\right\rangle\left\langle e_{1}\right| e^{i \Delta t}\right)+\hbar \lambda_{2}\left(a^{\dagger}|g\rangle\left\langle e_{1}\left|e^{-i \Delta t}+a\right| e_{1}\right\rangle\langle g| e^{i \Delta t}\right)
$$

where, $\Delta=\omega_{0}-\omega$ is the detuning. Now, we suppose that the atom-field coupling is intensity dependent, expressed by $g(n)$, so the intensity-dependent Hamiltonian in the interaction picture is given by:

$$
\mathcal{H}_{I}=\hbar \lambda_{1}\left(R^{\dagger}\left|e_{1}\right\rangle\left\langle e_{2}\left|e^{-i \Delta t}+R\right| e_{2}\right\rangle\left\langle e_{1}\right| e^{i \Delta t}\right)+\hbar \lambda_{2}\left(R^{\dagger}|g\rangle\left\langle e_{1}\left|e^{-i \Delta t}+R\right| e_{1}\right\rangle\langle g| e^{i \Delta t}\right)
$$

where $R=a g(n), R^{\dagger}=g(n) a^{\dagger}$ and $g(n)$ describes the intensity-dependent coupling between atom and field. Notice that, $R$ and $R^{\dagger}$ in (3) have the same structure and also meaning of $A$ and $A^{\dagger}$ were described at the beginning of this section, except that a different nonlinearity function is considered. Our different notation is only for the distinction between the nonlinearity of the initial state of the field and the intensity dependent coupling function. We have assumed that $g(n)$ is a real well-defined function with no singularity. The Hamiltonian in (5) plays a crucial role in determining the subsequent dynamics of the quantum states of a variety of (three-level) atom-field systems, some of them will be considered in the continuation of the paper.

\section{Atom-field state vector}

Let the atom be initially in the first excited state $\left|e_{1}\right\rangle$ and the cavity field is prepared in a generalized CS with a nonlinear CS as given in (11). The state of the atom-field system at $t=0$ can be expressed as:

$$
|\Psi(t=0)\rangle=\left|e_{1}\right\rangle \otimes|\alpha, f\rangle=\sum_{n=0}^{\infty} C_{n}\left|e_{1}, n\right\rangle,
$$

where $C_{n}$ is determined as the expansion coefficients of the states in (1), according to the initial CS has been chosen. A useful approach to the atom-field interaction problem may be found in the interaction picture 21. The Schrödinger representation of a state vector $|\Psi(t)\rangle$, in terms of its interaction picture representation $\left|\Psi_{I}(t)\right\rangle$, is given by $|\Psi(t)\rangle=$ $U_{0}(t)\left|\Psi_{I}(t)\right\rangle$, where $U_{0}(t)=\exp \left(\frac{-i H_{0} t}{\hbar}\right)$. Also, $\left|\Psi_{I}(t)\right\rangle$ can be obtained from $i \hbar \frac{\partial}{\partial t}\left|\Psi_{I}(t)\right\rangle=$ $\mathcal{H}_{I}\left|\Psi_{I}(t)\right\rangle$. Consequently, by using the above relations, one can straightforwardly find the time evolution of the state vector of the coupled atom-field system in the resonance 
condition $\Delta=0$ as follows:

$$
\begin{aligned}
|\Psi(t)\rangle & =\sum_{n=0}^{\infty} e^{-i \omega_{0}\left(S_{z}+n\right) t} C_{n} \\
& \times \quad\left\{\cos \left(\sqrt{n g^{2}(n)+\beta^{2}(n+1) g^{2}(n+1)} \tau\right)\left|e_{1}, n\right\rangle\right. \\
& -i \quad \frac{\beta \sqrt{n+1} g(n+1)}{\sqrt{n g^{2}(n)+\beta^{2}(n+1) g^{2}(n+1)}} \\
& \left.\times \quad \sin \left(\sqrt{n g^{2}(n)+\beta^{2}(n+1) g^{2}(n+1)} \tau\right)|g, n+1\rangle\right\} \\
& -i \sum_{n=1}^{\infty} e^{-i \omega_{0}\left(S_{z}+n\right) t} C_{n} \frac{\sqrt{n} g(n)}{\sqrt{n g^{2}(n)+\beta^{2}(n+1) g^{2}(n+1)}} \\
& \times \sin \left(\sqrt{n g^{2}(n)+\beta^{2}(n+1) g^{2}(n+1)} \tau\right)\left|e_{2}, n-1\right\rangle,
\end{aligned}
$$

where $\beta \equiv \lambda_{2} / \lambda_{1}$ and $\tau \equiv \lambda_{1} t$. We will call $\tau$ which is the scaled time briefly as time. It is worth noticing that $n$ which is appeared in the exponential functions in (7) has the operational role (number operator), the same as the role of $S_{z}$. Anyway, this solution is very general and gives the time evolution of a system that is involved a single-mode cavity field, has been prepared in a generalized CS, coupled with an equidistant threelevel atom in a cascade configuration with different dipole moment matrix transition elements between the adjacent levels $\left(\lambda_{1} \neq \lambda_{2}\right)$. Two specific cases can be recovered: (1) when $\lambda_{2} \rightarrow 0(\beta \rightarrow 0)$, it is equivalent to the single two-level atom; and (2) when $\lambda_{2} \rightarrow \lambda_{1}(\beta \rightarrow 1)$ it corresponds to an equal dipole moment matrix transition elements between the adjacent levels. This case is indeed equivalent to a pair of indistinguishable two-level atoms [22]. The states of the atomic pair can be described in the three-level states representation: (i) the ground state is equivalent to the case in which both atoms of the pair are in the ground state; (ii) the first exited state describes the case in which one atom of the pair is in the ground state and another atom is in the exited state, and (iii) in the second exited state, both atoms of the pair are in the exited state. In addition to the generality of our presented formalism, another advantage of our formalism is that it contains two distinct nonlinearity functions, i.e., $f(n)$ corresponding to initial state of the field and $g(n)$ which determines the intensity-dependent coupling between atom and field. So, we can easily recover the results in [20], if we take $f(n)=1 / \sqrt{n}$ and $g(n)=\sqrt{n}$ and also in [18], if we take $f(n)=1 / \sqrt{n}, g(n)=\sqrt{n}$ with $\beta=0$, as some special cases. Also, we can work with other different possibilities, especially the case $g(n)=f(n)=1$ and $g(n)=f(n) \neq 1$, investigate the output results and compare with [20]. 


\section{Quantum statistics and non-classicality of the in- troduced state}

After determining the state vector of the atom-field system at any arbitrary time obtained in (77), we are able to investigate mean photon number, atomic population inversion, Mandel parameter and squeezing parameters as some non-classicality criteria.

\subsection{Mean photon number}

Using the explicit form of atom-field system $|\Psi(t)\rangle$ in (7), the mean photon number can be readily found for any system, with arbitrary $f(n)$ and $g(n)$ as follows:

$$
\begin{aligned}
\langle n\rangle & =\sum_{n=0}^{\infty}\left|C_{n}\right|^{2} n \cos ^{2}\left(\sqrt{n g^{2}(n)+\beta^{2}(n+1) g^{2}(n+1)} \tau\right) \\
& +\sum_{n=0}^{\infty}\left|C_{n}\right|^{2} \sin ^{2}\left(\sqrt{n g^{2}(n)+\beta^{2}(n+1) g^{2}(n+1)} \tau\right) \\
& \times \frac{n(n-1) g^{2}(n)+\beta^{2}(n+1)^{2} g^{2}(n+1)}{n g^{2}(n)+\beta^{2}(n+1) g^{2}(n+1)} .
\end{aligned}
$$

\subsection{Atomic population inversion}

Atomic population inversion, as the expectation value of $S_{z}$, is defined as

$$
\left\langle S_{z}\right\rangle=\left\langle\Psi(t)\left|S_{z}\right| \Psi(t)\right\rangle=\left|\left\langle e_{2} \mid \Psi(t)\right\rangle\right|^{2}-|\langle g \mid \Psi(t)\rangle|^{2},
$$

where $\left|\left\langle e_{2} \mid \Psi(t)\right\rangle\right|^{2}$ and $|\langle g \mid \Psi(t)\rangle|^{2}$ are the probabilities of the presence of the atom in $\left|e_{2}\right\rangle$ and $|g\rangle$ states, respectively. With the help of $|\Psi(t)\rangle$ in (17), $\left\langle S_{z}\right\rangle$ can be written as:

$$
\begin{aligned}
\left\langle S_{z}\right\rangle & =\sum_{n=0}^{\infty}\left|C_{n}\right|^{2} \sin ^{2}\left(\sqrt{n g^{2}(n)+\beta^{2}(n+1) g^{2}(n+1)} \tau\right) \\
& \times \frac{n g^{2}(n)-\beta^{2}(n+1) g^{2}(n+1)}{n g^{2}(n)+\beta^{2}(n+1) g^{2}(n+1)} .
\end{aligned}
$$

As a special case, in the absence of the radiation field inside the cavity, namely, the cavity field being in the vacuum state $(n=0)$, the quantity $\left\langle S_{z}\right\rangle$ is given by:

$$
\left\langle S_{z}\right\rangle_{n=0}=-\left|C_{0}\right|^{2} \sin ^{2}(\beta g(1) \tau) \text {. }
$$

Recall that, the atom is initially in $\left|e_{1}\right\rangle$. Therefore, in the absence of a driving field, the atom in the lower state $\left|e_{1}\right\rangle$, cannot excite to the upper state $\left|e_{2}\right\rangle$, so $\left(\left|\left\langle e_{2} \mid \Psi(t)\right\rangle\right|^{2}\right)_{n=0}=0$ and from (9) one has

$$
\left\langle S_{z}\right\rangle_{n=0}=-\left(|\langle g \mid \Psi(t)\rangle|^{2}\right)_{n=0}
$$


By the comparison of (11) and (12), one can arrive at $\left(|\langle g \mid \Psi(t)\rangle|^{2}\right)_{n=0}=\left|C_{0}\right|^{2} \sin ^{2}(\beta g(1) \tau)$, which means that in the fully quantum theory of radiation, transition from the upper state to the lower state in the vacuum of the field becomes possible, known as the spontaneous emission. It is to be noted that this result cannot be predicted by semiclassical radiation theory.

\subsection{Mandel's $Q$-parameter}

To examine the statistics of the states, Mandel's $Q$-parameter is widely used, characterizes the quantum statistics of the states inside the cavity. This parameter has been defined as $Q=\frac{\left\langle n^{2}\right\rangle-\langle n\rangle^{2}}{\langle n\rangle}-1$ [23], where $\langle n\rangle$ obtained in (8) and $\left\langle n^{2}\right\rangle$ may be calculated as follows:

$$
\begin{aligned}
\left\langle n^{2}\right\rangle & =\sum_{n=0}^{\infty}\left|C_{n}\right|^{2}\left\{n^{2} \cos ^{2}\left(\sqrt{n g^{2}(n)+\beta^{2}(n+1) g^{2}(n+1)} \tau\right)\right. \\
& +\frac{n(n-1)^{2} g^{2}(n)+\beta^{2}(n+1)^{3} g^{2}(n+1)}{n g^{2}(n)+\beta^{2}(n+1) g^{2}(n+1)} \\
& \left.\times \sin ^{2}\left(\sqrt{n g^{2}(n)+\beta^{2}(n+1) g^{2}(n+1)} \tau\right)\right\} .
\end{aligned}
$$

The Mandel's $Q$-parameter obviously depends on the particular choice of $f(n)$ and $g(n)$. It is well-known that $Q$ is positive for classical states (super-Poissonian), negative for non-classical states (sub-Poissonian) and vanishes for canonical CSs (Poissonian).

\subsection{Squeezing parameters}

Also, we will investigate the squeezing properties of the quadratures of the field. For this purpose, we introduce field quadratures as $X_{1}=\frac{\mathcal{A}+\mathcal{A}^{\dagger}}{2}, X_{2}=\frac{\mathcal{A}-\mathcal{A}^{\dagger}}{2 i}$, where $\mathcal{A}$ and $\mathcal{A}^{\dagger}$ are the operators $\mathcal{A}=a e^{i \omega t}, \mathcal{A}^{\dagger}=a^{\dagger} e^{-i \omega t}$. To study the squeezing properties, we introduce the squeezing parameters:

$$
S_{j}(\tau)=4\left\langle\left(\triangle X_{j}\right)^{2}\right\rangle-1
$$

where $S_{j}(\tau)$ corresponds to squeezing effect in $X_{j}$ and satisfies the inequalities $-1<$ $S_{j}(\tau)<0$. Obviously, to preserve the Heisenberg uncertainty relation, when $S_{1}\left(S_{2}\right)$ is negative, $S_{2}\left(S_{1}\right)$ should be positive. To calculate the parameters $S_{j}(\tau)$ numerically, one has to find the mean values of the operators: $a, a^{2}, a^{\dagger}$ and $a^{\dagger^{2}}$. The following results are easily obtained:

$$
\langle a\rangle=e^{-i(\omega t-\varphi)} B_{1}(\tau),\left\langle a^{2}\right\rangle=e^{-2 i(\omega t-\varphi)} B_{2}(\tau)
$$


where

$$
\begin{aligned}
B_{1}(\tau) & =e^{-i \varphi} \sum_{n=0}^{\infty} C_{n}^{*} C_{n+1}\{\sqrt{n+1} \\
& \times \cos \left(\sqrt{n g^{2}(n)+\beta^{2}(n+1) g^{2}(n+1)} \tau\right) \\
& \times \cos \left(\sqrt{(n+1) g^{2}(n+1)+\beta^{2}(n+2) g^{2}(n+2)} \tau\right) \\
& +\frac{\sin \left(\sqrt{n g^{2}(n)+\beta^{2}(n+1) g^{2}(n+1)} \tau\right)}{\sqrt{n g^{2}(n)+\beta^{2}(n+1) g^{2}(n+1)}} \\
& \times \frac{\sin \left(\sqrt{(n+1) g^{2}(n+1)+\beta^{2}(n+2) g^{2}(n+2)} \tau\right)}{\sqrt{(n+1) g^{2}(n+1)+\beta^{2}(n+2) g^{2}(n+2)}} \\
& \left.\times \sqrt{n+1} g(n+1)\left[n g(n)+\beta^{2}(n+2) g(n+2)\right]\right\} \\
B_{2}(\tau) & =e^{-2 i \varphi} \sum_{n=0}^{\infty} C_{n}^{*} C_{n+2}\{\sqrt{n+1} \sqrt{n+2} \\
& \times \cos \left(\sqrt{n g^{2}(n)+\beta^{2}(n+1) g^{2}(n+1)} \tau\right) \\
& \times \cos \left(\sqrt{(n+2) g^{2}(n+2)+\beta^{2}(n+3) g^{2}(n+3)} \tau\right) \\
& +\frac{\sin \left(\sqrt{n g^{2}(n)+\beta^{2}(n+1) g^{2}(n+1)} \tau\right)}{\sqrt{n g^{2}(n)+\beta^{2}(n+1) g^{2}(n+1)}} \\
& \times \frac{\sin \left(\sqrt{(n+2) g^{2}(n+2)+\beta^{2}(n+3) g^{2}(n+3)} \tau\right)}{\sqrt{(n+2) g^{2}(n+2)+\beta^{2}(n+3) g^{2}(n+3)}} \\
& \times \sqrt{n+1} \sqrt{n+2}[n g(n) g(n+2) \\
+ & \left.\left.\beta^{2}(n+3) g(n+1) g(n+3)\right]\right\} .
\end{aligned}
$$

Clearly, $\left\langle a^{\dagger}\right\rangle=\langle a\rangle^{\dagger}$ and $\left\langle a^{\dagger^{2}}\right\rangle=\left\langle a^{2}\right\rangle^{\dagger}$. It should be noticed that $B_{1}(\tau)$ and $B_{2}(\tau)$ are real values. Finally, using the above expressions we arrive at

$$
\begin{aligned}
& S_{1}(\tau)=2\left[B_{0}(\tau)-B_{2}(\tau)\right]+4 \cos ^{2}(\varphi)\left[B_{2}(\tau)-B_{1}^{2}(\tau)\right], \\
& S_{2}(\tau)=2\left[B_{0}(\tau)-B_{2}(\tau)\right]+4 \sin ^{2}(\varphi)\left[B_{2}(\tau)-B_{1}^{2}(\tau)\right],
\end{aligned}
$$

where we have set $B_{0}(\tau) \equiv\langle n\rangle$.

\section{Some physical realizations of the formalism}

In this section, we firstly consider the special simple case $f(n)=g(n)=1$ and then nonlinearity functions of CSs of $S U(1,1)$ group are considered. 


\subsection{The special case: $f(n)=g(n)=1$}

In this subsection it will be constructive to consider canonical coherent state as the initial state of the field and suppose that the coupling between (the three-level) atom and field be independent of intensity, namely $f(n)=g(n)=1$. The quantities that have been presented in section 4, are plotted versus time for this special case. From figures 2-4 it is seen that mean photon number, atomic population inversion and Mandel parameter have nearly complete collapses and revivals, at least at intermediate times. This quantities collapses to $\langle n\rangle \simeq 63.5,\left\langle S_{z}\right\rangle \simeq 0.5$ and $Q \simeq 0.01$, respectively. Atomic population inversion is positive. This means that the probability of the presence of the atom in $\left|e_{2}\right\rangle$ is greater than being in $|g\rangle$. Mandel parameter is negative in some time intervals, namely the state of the system possesses sub-Poissonian statistics. Squeezing parameters versus time is shown in Fig. 5. There is no squeezing in $X_{1}$ and nearly in $X_{2}$, too if one ignores the very weak squeezing that may be seen about $\tau=45$.

\subsection{Nonlinear CSs: CSs of $S U(1,1)$ group}

At this stage of the paper, as an example of initial nonlinear CS, we will consider the Holestein-Primakoff single-mode realization of $S U(1,1)$ Lie algebra. Before we proceed, it is worth noticing that, the equivalence of the discrete series representation of $S U(1,1)$ state space $\{|\kappa, n\rangle\}_{n=0}^{\infty}$, with $\kappa=\frac{1}{2}, 1, \frac{3}{2}, 2, \ldots$, and the harmonic oscillator Hilbert space $\{|n\rangle\}_{n=0}^{\infty}$ is illustrated in [24]. Based on this recognition, $S U(1,1)$ CSs have been well established as nonlinear CSs by Ali et al [25].

We briefly introduce the GP CSs of $S U(1,1)$ group (sometimes have been called Klauder-Perelomov CSs [2, 3]). These states [26] are defined in the interior of the unit disk in the complex plane, centered at the origin. The nonlinearity function corresponding to these states is deduced as $f_{G P}(n, \kappa)=1 / \sqrt{n+2 \kappa-1}$ [1, 11, 27]. In this subsection, we first take $f(n)=g(n)=f_{G P}(n, \kappa)$ in our further numerical calculations. Different quantities mentioned in section 4 associated to the atom-field state (7), have been plotted versus time in figures 6-9 with fixed parameters $\kappa=3 / 2,|\alpha|=0.9$ and $\beta=0.01$. Figure 6 deals with the mean photon number. A typical fractional collapses and revivals are visible from the figure $6 \mathrm{a}$. This figure indicates that the envelope of the oscillations fractionally collapses to a fixed value $\simeq 12.3$ and as time goes on, the collapsed mean photon number is partly revived. The maximum amplitude of the oscillations occurred at $\tau=0$ decreases with time, and the duration of the oscillations varies, irregularly. Our aim for showing the figure in a short time interval is to explain the details of the variation of mean photon number in a more apparent fashion, particularly in relation to next figure. Atomic population inversion is shown in figure 7 . The positivity of this quantity at all time means that the probability of the presence of the atom in the state $\left|e_{2}\right\rangle$ is larger than the probability of being in the state $|g\rangle$. Similar to mean photon number, fractional collapses and revivals are observable from figure $7 \mathrm{a}$. It is to be noted that, in 
this case the atomic population inversion collapses to a fixed value $\simeq 0.5$. Figures $6 \mathrm{~b}$ and $7 \mathrm{~b}$ are the same as figures $6 \mathrm{a}$ and $7 \mathrm{a}$, respectively, with the same chosen parameters, except that the interval of time are restricted to 20 . A comparison between the figures $6 \mathrm{~b}$ and $7 \mathrm{~b}$ shows that they both obey nearly the same general pattern and makes one sure that the variations of mean photon number and the atomic population inversion are in opposite directions, as is expected. Figure 8 displays Mandel parameter as a function of time. The supper-Poissonian behaviour is observed from the figure, together with a (typical) collapses and revivals. This phenomenon occurs for the system under consideration, although not in a clear and regular manner. Indeed, Mandel parameter oscillates such drastically with time that the quasi-chaotic behaviour is revealed. In figure 9 the curves of squeezing parameters $S_{1}$ and $S_{2}$ are shown for $\varphi=\pi / 2$. Only the negativity of $S_{1}$, which indicates squeezing effect in $X_{1}$ quadrature, is revealed in a finite interval of time.

At this stage, in contrast to Buzek [18] and Koroli et al [20] that took $f(n)=$ $f_{G P}(n, 1 / 2)=1 / \sqrt{n}$ and $g(n)=\sqrt{n}$, we will set $f_{B G}(n)=g(n)=\sqrt{n}$ and follow our numerical calculations. The nonlinearity function $\sqrt{n}$ can be associated with a particular case of the BG state when one chooses $\kappa=\frac{1}{2}$. These states are defined in the whole of the complex plane. We emphasize that as in the GP CSs, we consider the HolesteinPrimakoff realization of $S U(1,1)$ Lie algebra of BG states. Barut-Girardello (BG) CSs of $S U(1,1)$ group [28] are established as the dual pair of GP CSs [25]. The nonlinearity function of these states is obtained as $f_{B G}(n, \kappa)=\sqrt{n+2 \kappa-1}$ [11, 25, 27]. It is easy to check that the operators $K_{-, B G}=a f_{B G}(n), K_{+, B G}=f_{B G}(n) a^{\dagger}, K_{0, B G}=\frac{1}{2}\left[K_{-, B G}, K_{+, B G}\right]$ satisfy the commutation relations $\left[K_{-, B G}, K_{+, B G}\right]=2 K_{0, B G},\left[K_{0, B G}, K_{ \pm, B G}\right]= \pm K_{ \pm, B G}$. where $K_{0}=n+\kappa$.

Anyway, choosing $f(n)=g(n)=f_{B G}(n, 1 / 2)=\sqrt{n}$, our calculated results have been displayed in figures 10-13. The mean photon number and atomic population inversion versus time for the corresponding atom-field states have been shown in figures 10 and 11, respectively. In the range which the atomic population inversion gets negative values, the probability of the presence of the atom in $|g\rangle$ is indeed more than $\left|e_{2}\right\rangle$. Observing figures 10 and 11, it will be clear that, any increase (decrease) in population inversion is simultaneous with a decrease (increase) in mean photon number. Also, there are two distinct oscillatory behaviour in these two quantities, one (small) is within the other (large). This feature was not appeared in [20], when they took $f(n) \neq g(n)$ (figures 1 and 2 of Ref. [20]). While there are some jumps in the mean photon number (which simultaneously accompanied by a sudden decrease in the atomic population inversion), according to our results small jumps and decrease within great jumps and decrease will be revealed. It is worth mentioning that, we continued our numerical results (have not shown here) with calculating the mean photon number and atomic population inversion in a wide interval of time and observed that, for both values of the considered $\beta$, the quantities are nearly (not exactly) periodic. But, the period of time for the case $\beta=0.01$ is larger than the case $\beta=0.1$. In figure 12, the Mandel parameter has been displayed as 
a function of time. The results indicate that for $\beta=0.1$ this parameter is almost periodic, while its maximum amplitude decreases with time. The sub-Poissonian behaviour also occurs in wide intervals of time. For large times, full sub-Poissonian statistics will be revealed for such system, in both of the presented cases. It is worth noticing that, this non-classicality sign has not been observed in [20]. In figure 13, the curves of squeezing parameters repeated regularly, but not exactly. Only for small intervals of time, squeezing effect is occurred in $X_{1}$ or $X_{2}$ quadrature.

Also a specific case can be regarded, i.e., when $f(n)=1$, which means that the initial field is in the standard CS and the interaction between atom and field would be intensity-dependent. For instance, we consider $g(n)=f_{G P}(n, \kappa)=1 / \sqrt{n+2 \kappa-1}$. Our numerical results have been displayed in figures 14-17. In figure 14 the mean photon number for the atom-field state (7) associated with these particular functions is shown. Atomic population inversion is plotted versus time in figure 15. A careful observation on the figures 14, 15 leads one to conclude that, when the mean photon number of the field is increased (decreased) due to emission (absorbtion), the probability of the presence of the atom to be in the $|g\rangle\left(\left|e_{2}\right\rangle\right)$ state increases, consequently the atomic population inversion is reduced (increased). Figure 16 shows the Mandel parameter, which implies that it is negative in a wide range of time, so that sub-Poissonian behaviour occurs, repeatedly. As shown in figure 17, in small regions of time the squeezing effect is observed weakly in $X_{1}$ or $X_{2}$ quadratures, separately. Obviously, when $S_{1}\left(S_{2}\right)$ is negative, $S_{2}\left(S_{1}\right)$ have to be positive.

Summing up the above presented results, two remarkable points may be offered. Firstly, the represented numerical results plotted in figures 2-5 for the case $f(n)=g(n)=$ 1 show that the collapses and revivals occur nearly regularly (but not exactly) relative to the next ones which contain some kind of nonlinearities. Indeed, in the latter cases the chaotic behaviour will be revealed due to the presence of the nonlinearities. In general, the Mandel parameter and squeezing effect have not an exact regular periodicity, specifically relative to two-level atoms. In addition, as it is expected, the variations of mean photon number and the atomic population inversion are in opposite directions. As we have explained, this is consistence with the physics of the considered interaction. Also, the fractional collapses and revivals phenomenon, as a well-known non-classicality sign, is seen in the mean photon number, atomic population inversion and Mandel parameter in the two groups of figures 6-8. The negativity of Mandel parameter in a wide range of time is observed in figures 12 and 16. The latter effect, will become more important, if one recalls that the squeezing signature of the field is revealed only in a small finite interval of time (see figures 9, 13, 17). 


\section{Summary and concluding remarks}

In summary, we have considered the interaction of an equidistant three-level atom in a cascade configuration with different dipole moment matrix transition elements between the adjacent atomic levels, and a single-mode cavity field that is initially prepared in a generalized CS. It should be emphasized that, our formalism is presented in a very general regime, because it contains two nonlinearity functions: $f(n)$ which characterizes the initial state of the field and $g(n)$ which determines the intensity-dependent coupling between atom and field. Particularly, $f(n)=g(n)=1$ is equivalent to the case in which the initial state of the field is standard CS and the interaction between atom and field be independent of intensity. Interestingly, we have presented a closed analytical solution for such a non-trivial problem. Then, as some physical appearances of the proposed structure, we have investigated the mean photon number, atomic population inversion, Mandel parameter and squeezing parameters for GP and BG CSs of $S U(1,1)$ group as well as some special cases. A few points are remarkable, regarding the presented results.

- Unlike the reported work by Buzek in [18] which considered the two-level atom, we have not observed the exact regular periodicity of the squeezing parameters, in neither of the chosen nonlinearity functions. We also calculated the Mandel parameter and find that the exact periodicity which occurs in two-level atom violates. These phenomenon are consistence with the reported results in [20]. We investigated and examined this observations for various cases, either with the same nonlinearities $(f(n)=g(n))$ or with different nonlinearities $(f(n) \neq g(n))$.

- The variation of mean photon number and atomic population inversion are in opposite directions, which is an expected result, in view of the physics of the systems under the considered interaction. Also, for the special case $f_{G P}(n)=g(n)=$ $1 / \sqrt{n+2 \kappa-1}$ in the second group of figures, the fractional collapses and revivals phenomenon in the mean photon number and the atomic population inversion, as well as the Mandel parameter are new features of our proposal, may be highlighted.

- Our results confirm that only in the fully quantum theory of radiation, the spontaneous emission (transition from the upper state to the lower one) in the vacuum of the field becomes possible.

- Comparing the figures 2-5, represented the numerical results have been plotted for the case $f(n)=g(n)=1$, with next figures one can see that the collapses and revivals occur more regularly for the former ones relative to others which contain some kind of nonlinearities, either in nonlinearity function of initial state or the intensity-dependent coupling. Indeed, in the latter cases the chaotic behaviour will be revealed due to the presence of the nonlinearities. 
- Apart from these, the generality and at the same time the simplicity of our proposal allows one to apply it to other physical systems with known nonlinearity functions, for instance, center of mass motion of trapped ion [6], photon-added CSs [10, qCSs [7], deformed photon-added nonlinear coherent states [29] have been recently introduced by one of us and so on. These are straightforward tasks may be done elsewhere. On the other side, Roknizadeh et al introduced a Hamiltonian associated with a nonlinear oscillator system, based on action identity requirement of nonlinear CSs as follows: $H=A^{\dagger} A=n f^{2}(n)$ [11], upon which one obtains the eigenvalues $e_{n}=n f^{2}(n)$ or equivalently $f(n)=\sqrt{\frac{e_{n}}{n}}$. So, obviously the presented approach can be easily applied to such one-dimensional solvable systems, too.

\section{References}

[1] Ali S T, Antoine J-P and Gazeau J-P 2000 Coherent States, Wavelets and Their Generalization (Springer: New York)

[2] Klauder J R and Skagerstam B-S 1985 Coherent States, Applications in Physics and Mathematical Physics (Word Scientific: Singapore)

[3] Perelomov A 1986 Generalized Coherent States and Their Applications, Texts and Monographs in Physics (Spinger: Verlag)

[4] Klauder J R 2010 arXiv: 1008.4307 "On the Role of Coherent States in Quantum Foundations", ICQOQI Kiev Ukraine, Conference Proceedings

[5] Sixdeniers J-M, Penson K A and Klauder J R 2001, Int. J. Mod. Phys. B 15 4231; Darwish M 2005, Int. J. Mod. Phys. B 19 715; Eftekhari F and Tavassoly M K 2010 Int. J. Mod. Phys. A 253481

[6] Filho R L de Matos and Vogel W 1996 Phys. Rev. A. 544560

[7] Man'ko V I, Marmo G, Sudarshan E C G and Zaccaria F 1997 Phys. Scr. 55528

[8] Roy B 1998 Phys. Lett. A. 249 25; Roy B, Roy P 2000 J. Opt. B: Quantum Semiclass. Opt. 265

[9] Sivakumar S 2000 J. Opt. B: Quantum Semiclass. Opt. 2 R61

[10] Sivakumar S 1999 J. Phys. A: Math. Gen. 323441

[11] Roknizadeh R and Tavassoly M K 2004 J. Phys. A: Math. Gen. 378111

[12] Honarasa G R, Tavassoly M K and Hatami M 2009 Opt. Commun. 282 2192; ibid 2009 Phys. Lett. A. 3733931 
[13] Leon-Montiel R De J and Moya-Cessa H 2001 Int. J. Quant. Inform. 91 349; Naderi M H, Soltanolkotabi M and Roknizadeh R 2009 Int. J. Mod. Phys. A 241963

[14] Jaynes E T and Cummings F W 1963 Proc. IEEE. 5189

[15] Sivakumar S 2004 Int. J. Theor. Phys. 43 2405; Daoud M and Douari J 2003 Int. J. Mod. Phys. B 172473.

[16] Marchiolli M A, Physica A 2003319 331; Abdel-Aty M, Physica A 2002313 471; Abdel-Khalek S Physica A 2008387779

[17] Barzanjeh Sh, Naderi M H and Soltanolkotabi M 2010 J. Phys. A: Math. Theor. 43 375304

[18] Buzek V 1989 Phys. Rev. A. 393196

[19] Gilmore R 1974 Ann. Phys. NY 74 391; Perelomov A M 1972 Commun. Math. Phys. 26222

[20] Koroli V I and Zalamai V V 2009 J. Phys. B: At. Mol. Opt. Phys. 42 035505; ibid 2009 Int. J. Quant. Inform. 7179

[21] Scully M O and Zubairy M S Quantum Optics Cambridge UP, London (1997)

[22] Koroli V I 2007 Int. J. Quant. Inform. 5199

[23] Mandel L 1979 Opt. Lett. 4205

[24] Brif C 1995 Class. Quantum Grav. 12803

[25] Ali S T, Roknizadeh R and Tavassoly M K 2004 J. Math. Phys. 374407

[26] Brif C 1997 Int. J. Theor. Phys. 361651

[27] Roknizadeh R and Tavassoly M K 2005 J. Math. Phys. 46042110

[28] Barut A O and Girardello L 1971 Commun. Math. Phys. 2141

[29] Safaeian O and Tavassoly M K 2011 J. Phys. A: Math. Theor. 44225301 


\section{FIGURE CAPTIONS:}

Figure 1 Schematic diagram of the cosidered three-level atom.

Figure 2 The time variation of $\langle n\rangle$ with $|\alpha|=8$ and $\beta=0.01$ for $f(n)=g(n)=1$.

Figure 3 The time variation of $\left\langle S_{z}\right\rangle$ with $|\alpha|=8$ and $\beta=0.01$ for $f(n)=g(n)=1$.

Figure 4 The time variation of $Q$ with $|\alpha|=8$ and $\beta=0.01$ for $f(n)=g(n)=1$.

Figure 5 The time variation of $S_{1}$ (the solid curve) and $S_{2}$ (the dashed curve), with $|\alpha|=8, \beta=0.01$ and $\varphi=\pi / 2$ for $f(n)=g(n)=1$.

Figure 6 The time variation of $\langle n\rangle$ for different intervals of $\tau$, with $\kappa=3 / 2$ with $|\alpha|=0.9$ and $\beta=0.01$ for $f(n)_{G P}=g_{G P}(n)=1 / \sqrt{n+2 \kappa-1}$.

Figure 7 The time variation of $\left\langle S_{z}\right\rangle$ for different intervals of $\tau$, with $\kappa=3 / 2$ with $|\alpha|=0.9$ and $\beta=0.01$ for $f_{G P}(n)=g_{G P}(n)=1 / \sqrt{n+2 \kappa-1}$.

Figure 8 The time variation of $Q$ for $\kappa=3 / 2$ with $|\alpha|=0.9$ and $\beta=0.01$ for $f_{G P}(n)=g_{G P}(n)=1 / \sqrt{n+2 \kappa-1}$.

Figure 9 The time variation of $S_{1}$ (the solid curve) and $S_{2}$ (the dashed curve), for $\kappa=3 / 2$ with $|\alpha|=0.9, \beta=0.01$ and $\varphi=\pi / 2$ for $f_{G P}(n)=g_{G P}(n)=$ $1 / \sqrt{n+2 \kappa-1}$.

Figure 10 The time variation of $\langle n\rangle$ for $\beta=0.1$ (the solid curve) and $\beta=0.01$ (the dashed curve) with $|\alpha|=0.6$ for $f(n)=g(n)=\sqrt{n}$.

Figure 11 The time variation of $\left\langle S_{z}\right\rangle$ for $\beta=0.1$ (the solid curve) and $\beta=0.01$ (the dashed curve) with $|\alpha|=0.6$ for $f(n)=g(n)=\sqrt{n}$.

Figure 12 The time variation of $Q$ for $\beta=0.1$ (the solid curve) and $\beta=0.01$ (the dashed curve) with $|\alpha|=0.6$ for $f(n)=g(n)=\sqrt{n}$.

Figure 13 The time variation of $S_{1}$ (the solid curve) and $S_{2}$ (the dashed curve), with $|\alpha|=0.6, \beta=0.1$ and $\varphi=\pi / 2$ for $f(n)=g(n)=\sqrt{n}$.

Figure 14 The time variation of $\langle n\rangle$ for $\kappa=2$ (the solid curve) and $\kappa=4$ (the dashed curve) with $|\alpha|=0.5$ and $\beta=0.1$ for $f(n)=1, g_{G P}(n)=1 / \sqrt{n+2 \kappa-1}$.

Figure 15 The time variation of $\left\langle S_{z}\right\rangle$ for $\kappa=2$ (the solid curve) and $\kappa=4$ (the dashed curve) with $|\alpha|=0.5$ and $\beta=0.1$ for $f(n)=1, g_{G P}(n)=1 / \sqrt{n+2 \kappa-1}$.

Figure 16 The time variation of $Q$ for $\kappa=2$ (the solid curve) and $\kappa=4$ (the dashed curve) with $|\alpha|=0.5$ and $\beta=0.1$ for $f(n)=1, g_{G P}(n)=1 / \sqrt{n+2 \kappa-1}$.

Figure 17 The time variation of $S_{1}$ (the solid curve) and $S_{2}$ (the dashed curve), for $\kappa=2$ with $|\alpha|=0.5, \beta=0.1$ and $\varphi=\pi / 2$ for $f(n)=1, g_{G P}(n)=1 / \sqrt{n+2 \kappa-1}$. 